\title{
Performance Analysis of Machine Repair System with Balking, Reneging, Multiple Working Vacations and Two Removable Servers Operating under the Triadic $(0, Q, N, M)$ Policy
}

\author{
Teketel Ketema \\ Department of Mathematics Mekdela Amba University, Wollo, Ethiopia
}

\begin{abstract}
The main purpose of this paper is to investigate the performance analysis of $M / M / 2$ machine repair problem with impatient customers. A population size of $L$ operating machines is considered and the failed machines are assumed to be repaired under the multiple working vacations and triadic $(0, Q, N, M)$ policy. As soon as the system becomes empty, both servers leave for working vacations wherein only one of the two servers provide a service during the vacation. Steady state probabilities that describe the number of failed machines in system are derived and taken in closed form. Different performance measures of the system are developed and analysed with numerical illustrations to investigate the reliability of the model.
\end{abstract}

Keywords: machine repair problem, multiple working vacations, triadic $(0, Q, N, M)$ Policy, balking, reneging, steady state probability

DOI: $10.7176 /$ ISDE/11-4-03

Publication date:August $31^{\text {st }} 2020$

\section{Introduction}

The interaction of human and machine with industrial plants and other dynamic technical systems is nowadays essential for the quality and efficiency of the performance. Whenever and wherever machines are there, they ought to fail and repairing of such machine is required. In doing so, a scientific study in the interaction of servers and customers is mandatory for best performance of the system. Servers may become practically unavailable for a period of time due to a variety of reasons. This period of unavailability in queueing theory aspect is called vacation.

It is not difficult to observe customers arriving at a non-empty queuing system and leaving without joining the system. This behaviour of customers is known as balking. Customers may balk due to various reasons. On the other side, even if a customer does not balk and joins a queuing system, it is possible that the customer gets impatient and departs from the system without having completed the act of receiving service. Such impatient behaviour is known as reneging. In machining system, both balking and reneging are done by the caretaker who is responsible for getting the failed machines repaired.

Ma and Zhao (2016) discussed a machine repair queueing model that involves balking and reneging. Chandra et al. (2017) studied a machining system with geometric reneging. Geometric reneging of a machining system is also studied by Shekhar (2017). Wang et al. (2015) studied a machine repair problem with balking and reneging with general inter-arrival and service time distribution.

Chen et al. (2016) dealt with the reliability and sensitivity analysis of a machine repair system with warm standbys and an unreliable server having multiple-vacation wherein Laplace transform technique is used to derive the reliability function and the mean time to system failure. Jain and Meena (2018) applied a vacation model for a Markovian machine repair model. He et al. (2019) discussed a machine repair problem with a single working vacation. Jain et al. (2016) investigated a machine repair problem under $N$-policy wherein servers share their repair job simultaneously among all the failed machines that have joined the system for repair. Chen and Wang (2018) also analysed a reliability of a retrial machine repair problem with warm standbys and a single server with N-policy.

Sharma (2016) deals with machine interference problem with additional repairman and warm standby under Bernoulli vacation schedule in which threshold N-policy is considered for controlling the vacation period of repairman. Kumar (2018) applied $F$-policy (which is almost similar to the $N$ - policy) to the machine repair problems that has two unreliable servers and the provision of warm standbys. Ojobor and Ogini (2016) studied machine interference problem with reliable server under multiple vacations policy is considered. Meena et al. (2019) analysed a vacation model for a multi-component machine repair wherein fuzzy and harmony search optimization are applied to transform the machine repair model from crisp to fuzzy environment. A machining system with multiple vacations and heterogeneous servers is discussed by Ahuja and Jain (2019).

In this paper, impatient customers of a machine repair system with multiple working vacations and triadic $(0, Q, N, M)$ policy is considered for the first time. The definition of triadic $(0, Q, N, M)$ policy and multiple working vacations for the present model are defined in the sequel.

Whenever there are no failed machines in the system, both servers are turned off temporarily, and may not be reactivated until certain conditions are satisfied. Initially, it is supposed that both servers are on working vacation. 
When the number of failed machines in the system waiting for service reaches a specific quantity $N$, which is a decision variable, one of the two servers will be active instantly. At a later time, when the number of failed machines waiting for service increases to another specific quantity $M$, where $(N<M)$, then the remaining server will also be active immediately. However, when the number of failed machines in the system decreases to $Q$, where $(1 \leq Q<N)$, while both servers are active simultaneously, the server just finishing a service will be removed from the system at that time. Furthermore, when the number of failed machines in the system reaches to zero while one server is working, both servers will take a working vacation.

During working vacation, only one of the two servers will be active and serve the arriving failed machines at a different rate, generally lower than the regular service rate. At a working vacation completion epoch, if the system size is $N$ or above, then both servers will switch to a regular busy period and start working under the triadic $(0, Q, N, M)$ policy; otherwise, they will take another working vacations as often as necessary until the system size is $N$ or above at a vacation termination epoch.

\section{Description of the model}

A working vacation queue with two removable servers that operate under a triadic $(0, Q, N, M)$ policy is considered. The population size and system capacity are assumed to be finite with number $L$. Each of the operating machines fails independently of the state of the other. Whenever an operating machine fails, it immediately joins the system for repair. The inter-arrival time for the failed machines is assumed to be exponentially distributed with rate $\lambda$.

The arriving failed machines are assumed to form a single queue and the first-come-first-served queue discipline is implemented, as a result, the one at the head of the waiting line gets first into a service. The failed machines in the queue will get the service in two periods namely regular busy period and working vacation period. During the regular busy period, service is provided by two homogeneous servers each with service rate $\mu$ and exponentially distributed service time under the triadic $(0, Q, N, M)$ policy. Whereas only one of the two servers will provide a service during working vacation period. Vacation duration and service rate during the vacation are assumed to be exponentially distributed with rates $\emptyset$ and $\delta(\delta<\mu)$ respectively.

If the joined failed machine has to wait for service longer than its expectation, it may renege. The waiting time of the failed machine before reneging is assumed to be exponentially distributed with parameter $\varphi$ with the assumption that the arrival of a failed machine and renege of an impatient failed machine are independent. The average reneging rate when there are $n$ failed machines in the system is given by $r(n)=(n-1) \varphi, 1 \leq n \leq L$.

When the newly arrived failed machine finds $n$ failed machines in the system, then it will decide either to join the queue with probability $b$ or balk with the probability $1-b_{n}$, where

$$
b_{n}= \begin{cases}1, & n=0 \\ b, & 1 \leq n \leq L-1\end{cases}
$$

\section{Analysis of the Model}

In steady state, the following probabilities are defined.

- $\quad P_{0, n} \equiv$ The probability that servers are on working vacation and there are $n$ failed machines in the system, where $n=0,1,2, \ldots, L$.

- $\quad P_{1, n} \equiv$ The probability that one server is active during the regular busy period and there are $n$ failed machines in the system, where $n=1,2, \ldots, M-1$.

- $\quad P_{2, n} \equiv$ The probability that both servers are active during the regular busy period and there are $n$ failed machines in the system, where $n=Q+1, Q+2, Q+3, \ldots, L$.

Referring to Figure 1, the following steady state probabilities are found.

$$
\begin{gathered}
{[L \lambda] P_{0,0}=\delta P_{0,1}+\mu P_{1,1},} \\
{[(L-n) \lambda b+\delta+(n-1) \varphi] P_{0, n}=[(L-n+1) \lambda b] P_{0, n-1}+[\delta+n \varphi] P_{0, n+1},} \\
1 \leq n \leq N-1, \\
{[(L-n) \lambda b+\delta+(n-1) \varphi+\emptyset] P_{0, n}=[(L-n+1) \lambda b] P_{0, n-1}+[\delta+n \varphi] P_{0, n+1},} \\
N \leq n \leq L-1, \\
{[\delta+(L-1) \varphi+\emptyset] P_{0, L}=[\lambda b] P_{0, L-1},} \\
{[(L-1) \lambda b+\mu] P_{1,1}=[\mu+\varphi] P_{1,2},} \\
{[(L-n) \lambda b+\mu+(n-1) \emptyset] P_{1, n}=[(L-n+1) \lambda b] P_{1, n-1}+[\mu+n \varphi] P_{1, n+1},} \\
2 \leq n \leq Q-1 \\
{[(L-Q) \lambda b+\mu+(Q-1) \varphi] P_{1, Q}=[(L-Q+1) \lambda b] P_{1, Q-1}+[\mu+Q \varphi] P_{1, Q+1},} \\
{[(L-n) \lambda b+\mu+(n-1) \varphi] P_{1, n}=[(L-n+1) \lambda b] P_{1, n-1}+[\mu+n \varphi] P_{1, n+1},} \\
Q+1 \leq n \leq N-1 \\
{[(L-n) \lambda b+\mu+(n-1) \varphi] P_{1, n}=[(L-n+1) \lambda b] P_{1, n-1}+[\mu+n \varphi] P_{1, n+1}+\emptyset P_{0, n},} \\
N \leq n \leq M-2,
\end{gathered}
$$




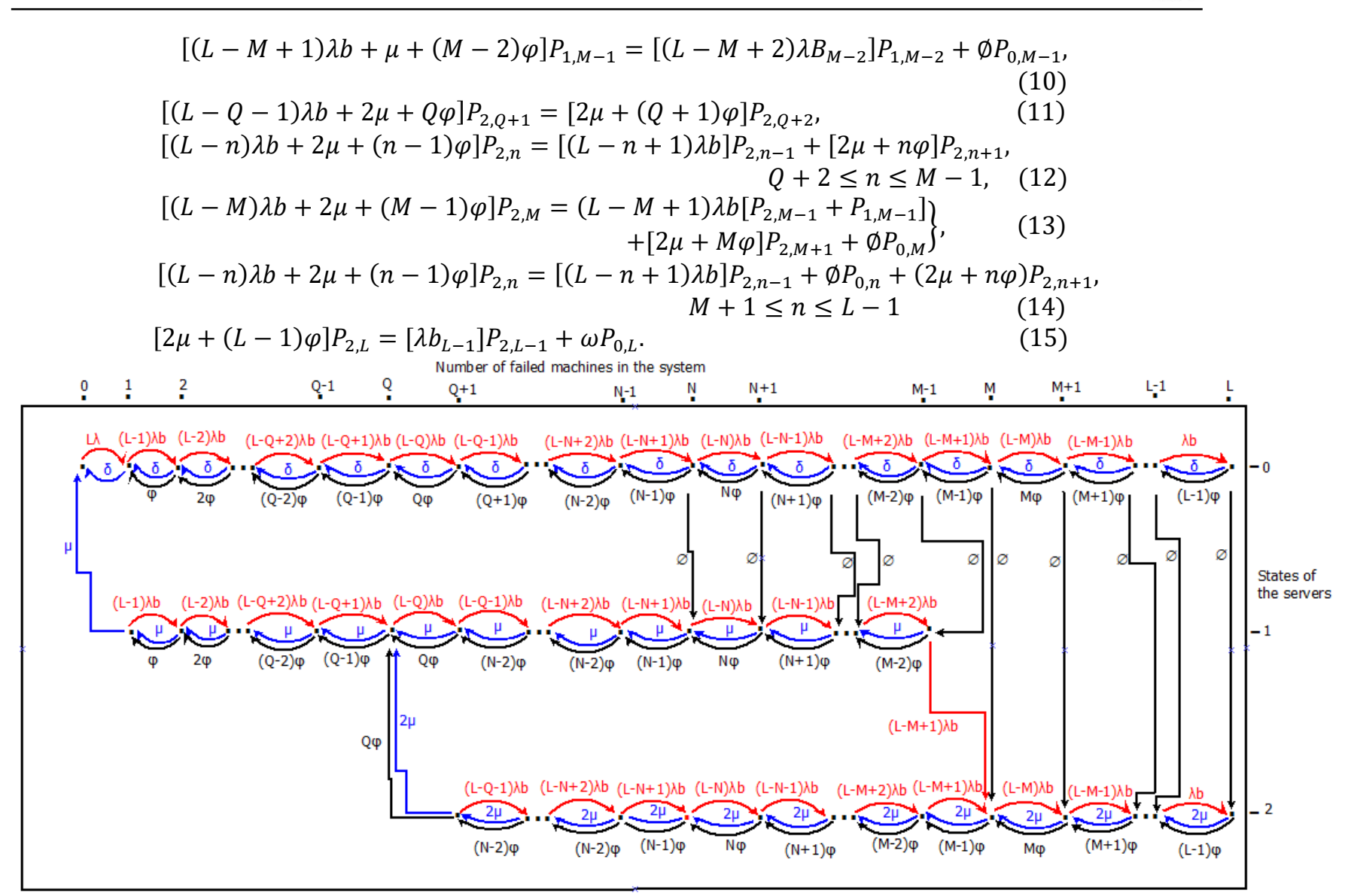

Figure 1. State-transition rate diagram for machine repair problem with balking, reneging, multiple working vacations and two removable servers operating under the triadic $(0, Q, N, M)$ policy

The solutions of the previously found steady state probabilities are found and put in neat closed-form by solving the system of equations (1) to (15) recursively.

where

$$
P_{0, L-1}=f_{L-1} P_{0, L}
$$

where

$$
\begin{aligned}
& f_{L-1}=\frac{\delta+(L-1) \varphi+\emptyset}{\lambda b}, f_{L}=1 . \\
& P_{0, n}=f_{n} P_{0, L},
\end{aligned}
$$

$$
\begin{gathered}
f_{n}=\left(\frac{(L-\mathrm{n}-1) \lambda b+\delta+n \varphi+\emptyset}{(L-n) \lambda b}\right) f_{n+1}-\left(\frac{\delta+(n+1) \varphi}{(L-n) \lambda b}\right) f_{n+2}, n=L-2, \ldots, N-1, \\
f_{n}=\left(\frac{(L-n-1) \lambda b+\delta+n \varphi}{(L-n) \lambda b}\right) f_{n+1}-\left(\frac{\delta+(n+1) \varphi}{(L-n) \lambda b}\right) f_{n+2}, n=N-2, \ldots, 0 . \\
P_{1, n}=g_{n} P_{0, L}, 1 \leq n \leq Q
\end{gathered}
$$

where

where

$$
\begin{aligned}
& g_{1}=\left(\frac{L \lambda}{\mu}\right) f_{0}-\left(\frac{\eta}{\mu}\right) f_{1}, g_{2}=\left(\frac{(L-1) \lambda b+\mu}{\mu+\varphi}\right) g_{1} \\
& g_{n}=\left(\frac{(L-n+1) \lambda b+\mu+(n-2) \varphi}{\mu+(n-1) \varphi}\right) g_{n-1}-\left(\frac{(L-n+2) \lambda b}{\mu+(i-1) \varphi}\right) g_{n-2}, n=3,4, \ldots, Q \\
& P_{1, Q+1}=g_{Q+1} P_{0, L}+h_{Q+1} P_{2, Q+1},
\end{aligned}
$$

$$
\begin{aligned}
& g_{Q+1}=\left(\frac{(L-Q) \lambda b+\mu+(Q-1) \varphi}{\mu+Q \varphi}\right) g_{Q}-\left(\frac{(L-Q+1) \lambda b}{\mu+Q \varphi}\right) g_{Q-1}, h_{n}=0,1 \leq n \leq Q, \\
& h_{Q+1}=-\left(\frac{2 \mu+Q \varphi}{\mu+Q \varphi}\right) . \\
& \quad P_{1, n}=g_{n} P_{0, L}+h_{n} P_{2, Q+1}, Q+2 \leq n \leq N
\end{aligned}
$$

where 
where

$$
\begin{gathered}
g_{n}=\left(\frac{(L-n+1) \lambda b+\mu+(n-2) \varphi}{\mu+(n-1) \varphi}\right) g_{n-1}-\left(\frac{(L-n+2) \lambda b}{\mu+(n-1) \varphi}\right) g_{n-2} \\
h_{n}=\left(\frac{(L-n+1) \lambda b+\mu+(n-2) \varphi}{\mu+(n-1) \varphi}\right) h_{n-1}-\left(\frac{(L-n+2) \lambda b}{\mu+(n-1) \varphi}\right) h_{n-2} . \\
P_{1, n}=g_{n} P_{0, L}+h_{n} P_{2, Q+1}, N+1 \leq n \leq M-1
\end{gathered}
$$

where

$$
\begin{aligned}
& \left.g_{n}=\left(\frac{(L-n+1) \lambda b+\mu+(n-2) \varphi}{\mu+(n-1) \varphi}\right) g_{n-1}-\left(\frac{(L-n+2) \lambda b}{\mu+(n-1) \varphi}\right) g_{n-2}\right\} \\
& \left.-\left(\frac{\emptyset}{\mu+(n-1) \varphi}\right) f_{n-1}\right\} \\
& h_{n}=\left(\frac{(L-n+1) \lambda b+\mu+(n-2) \varphi}{\mu+(n-1) \varphi}\right) h_{n-1}-\left(\frac{(L-n+2) \lambda b}{\mu+(n-1) \varphi}\right) h_{n-2} . \\
& P_{2, n}=k_{n} P_{0, L}+j_{n} P_{2, Q+1}, Q+1 \leq n \leq L,
\end{aligned}
$$

$k_{n}=0$ for $1 \leq n \leq M, j_{Q+1}=1$,

$$
\begin{aligned}
& j_{Q+2}=\left(\frac{(L-Q-1) \lambda b+2 \mu+Q \varphi}{2 \mu+(Q+1) \varphi}\right), \\
& j_{n}=\left(\frac{(L-n+1) \lambda b+2 \mu+(n-2) \varphi}{2 \mu+(n-1) \varphi}\right) j_{n-1}-\left(\frac{(L-n+2) \lambda b}{2 \mu+(n-1) \varphi}\right) j_{n-2}, Q+3 \leq n \leq M, \\
& j_{M+1}=\left(\frac{(L-M) \lambda b+2 \mu+(M-1) \varphi}{2 \mu+M \varphi}\right) j_{M}-\left(\frac{(L-M+1) \lambda b}{2 \mu+M \varphi}\right)\left(j_{M-1}+h_{M-1}\right), \\
& k_{M+1}=-\left(\left(\frac{(L-M+1) \lambda b}{2 \mu+M \varphi}\right) g_{M-1}+\left(\frac{\emptyset}{2 \mu+M \varphi}\right) f_{M}\right),
\end{aligned}
$$

for $M+2 \leq n \leq L$,

$$
\left.\begin{array}{c}
j_{n}=\left(\frac{(L-n+1) \lambda b+2 \mu+(n-2) \varphi}{2 \mu+(n-1) \varphi}\right) j_{n-1}-\left(\frac{(L-n+2) \lambda b}{2 \mu+(n-1) \varphi}\right) j_{n-2}, \\
\left.k_{n}=\left(\frac{(L-n+1) \lambda b+2 \mu+(n-2) \varphi}{2 \mu+(n-1) \varphi}\right) k_{n-1}-\left(\frac{(L-n+2) \lambda b}{2 \mu+(n-1) \varphi}\right) k_{n-2}\right) \\
-\left(\frac{\emptyset}{2 \mu+(n-1) \varphi}\right) f_{n-1}
\end{array}\right\} .
$$

Finally, from the normalization condition, $P_{2 . Q+1}$ is expressed as

$$
P_{2, Q+1}=\left[\sum_{i=0}^{L} f_{i} \frac{1}{\sigma}+\sum_{i=1}^{M-1}\left(g_{i} \frac{1}{\sigma}+h_{i}\right)+\sum_{i=Q+1}^{L}\left(k_{i} \frac{1}{\sigma}+j_{i}\right)\right]^{-1} .
$$

\section{Performance Measures of the Model}

Performance measures are the specific measurements of the quality of service. This is important to assess some well-defined parameters, which are designed at striking a good balance between customer satisfaction and economic considerations. Problems caused by queuing situations are often related to customer's dissatisfaction with service or may be the root cause of economic losses in a business. Analysis of the relevant performance measures of queuing models allows the cause of queuing issues to be identified and the impact of proposed changes to be assessed. The following system performance measures have been defined for the present machine repair model;

- $E[N] \equiv$ the expected number of failed machines in the system;

$$
E[N]=\sum_{n=1}^{L} n P_{0, n}+\sum_{n=1}^{M-1} n P_{1, n}+\sum_{n=Q+1}^{L} n P_{2, n}
$$

- $E[O] \equiv$ the expected number of operating machines in the system;

$$
E[O]=L-E[N]
$$

- $E[I] \equiv$ the expected number of idle servers in the system; 


$$
E[I]=2 P_{0,0}+\sum_{i=1}^{L} P_{0, n}+\sum_{i=1}^{M-1} P_{1, n}
$$

- $E\left[B_{0}\right] \equiv$ the expected number of one busy server during the working vacation period;

$$
E\left[B_{0}\right]=\sum_{n=1}^{L} P_{0, n}
$$

- $E\left[B_{1}\right] \equiv$ the expected number of one busy server during the regular busy period;

$$
E\left[B_{1}\right]=\sum_{n=1}^{M-1} P_{1, n}
$$

- $E\left[B_{2}\right] \equiv$ the expected number of two busy servers during the regular busy period;

$$
E\left[B_{2}\right]=\sum_{n=Q+1}^{L} 2 P_{2, n}
$$

- $\quad E_{Q} \equiv$ the expected queue length;

$$
E_{Q}=\sum_{n=1}^{L}(n-1) P_{0, n}+\sum_{n=1}^{M-1}(n-1) P_{1, n}+\sum_{n=Q+1}^{L}(n-2) P_{2, n}
$$

- $\quad M A \equiv$ Machine availability (the fraction of total time that the machines are working);

$$
M A=\frac{E[O]}{L}
$$

- $\quad O U \equiv$ Operative utilization (the fraction of busy servers);

$$
O U=\frac{E\left[B_{0}\right]+E\left[B_{1}\right]+E\left[B_{2}\right]}{2}
$$

- $\quad B R \equiv$ the average balking rate;

$$
\left.\begin{array}{rl}
B R=\sum_{n=1}^{L}(L-n) \lambda(1-b) P_{0, n} & +\sum_{n=1}^{M-1}(L-n) \lambda(1-b) P_{1, n} \\
& +\sum_{n=Q+1}^{L}(L-n) \lambda(1-b) P_{2, n}
\end{array}\right\}
$$

- $R R \equiv$ the average reneging rate

$$
R R=\sum_{n=1}^{L}(n-1) \varphi P_{0, n}+\sum_{n=1}^{M-1}(n-1) \varphi P_{1, n}+\sum_{n=Q+1}^{L}(n-1) \varphi P_{2, n} .
$$

\section{Numerical Illustrations}

This section presents some numerical results in the form of graphs, to validate the theoretical results obtained earlier. The parameters of the model are chosen to be $Q=3, N=15, M=23, L=30, \lambda=0.5, \mu=2.0, \delta=$ $0.8, \emptyset=1.0, \varphi=0.4$ and $b=0.5$, unless they are considered as variables or their values are mentioned in the respective figures. 


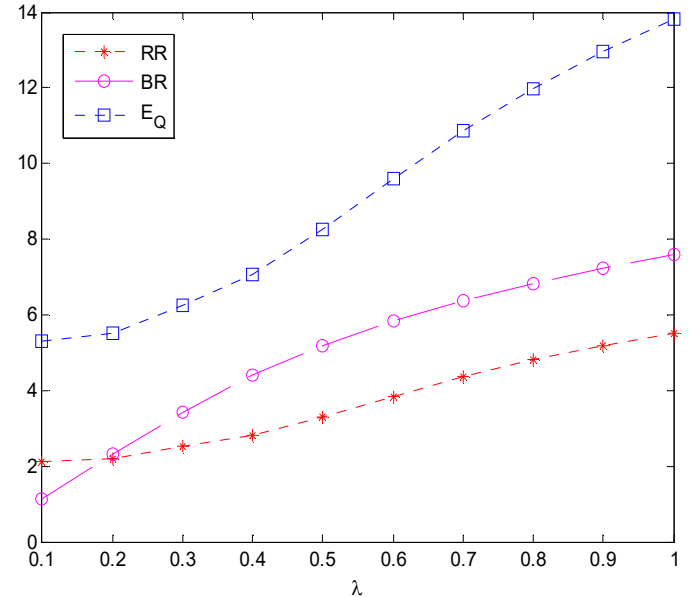

Figure 2. Effect of $\lambda$ on $R R, B R$ and $E_{Q}$

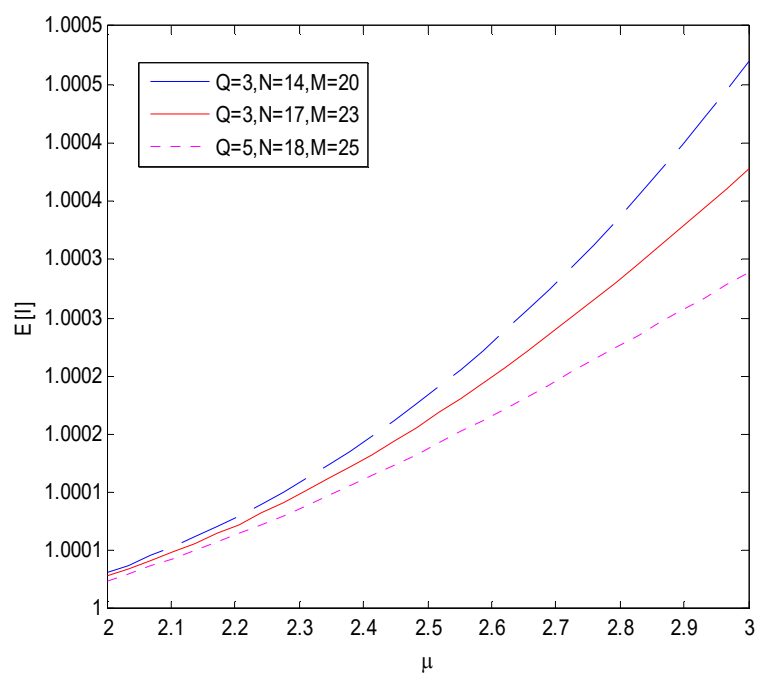

Figure 4. Efect of $\mu$ on $E[I]$

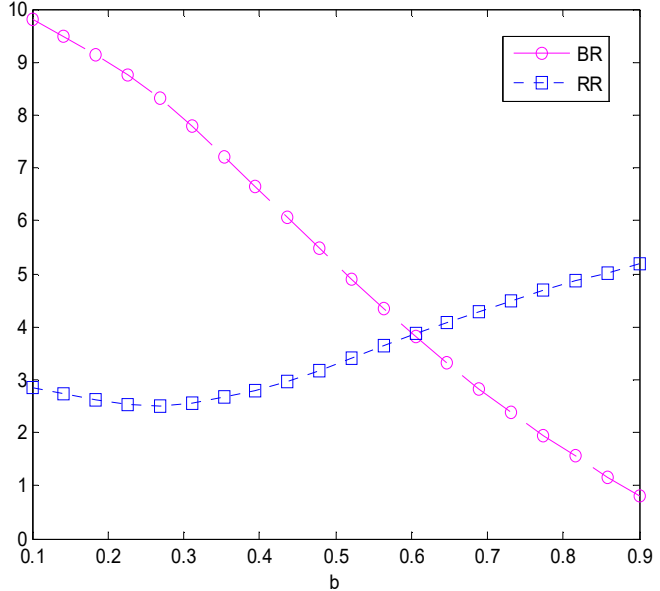

Figure 3. Effect of $b$ on $B R$ and $R R$

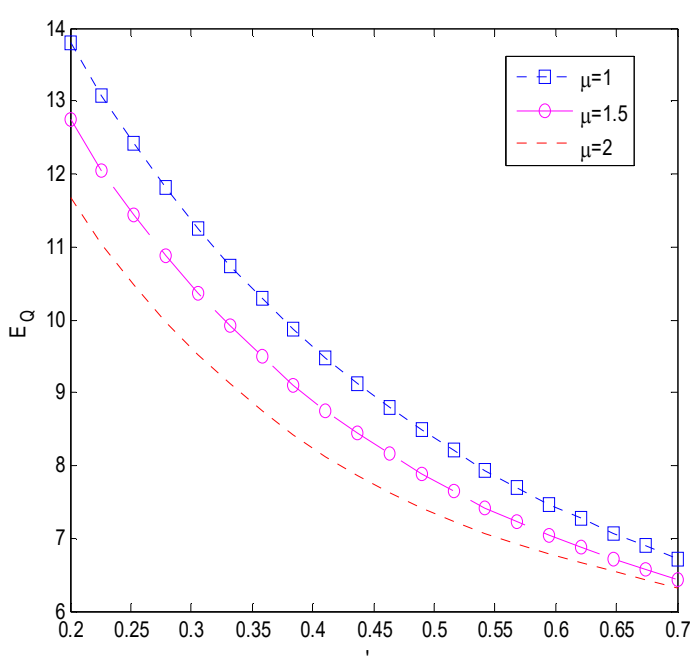

Figure 5. Effect of $\varphi$ on $E_{Q}$

Figure 2 shows that the increment of $\lambda$ results in an increment of balking and reneging rates and expected queue length as intuitively expected. The effect of $b$ on average balking and reneging rate is shown in Figure 3 . High customer intensity of joining the queue leads the queued failed machines to leave without service.

The impact of $\mu$ on expected number of idle server with different $Q, N \& M$ values are shown in Figure 4. It shows that for fixed $\mu$, the expected number of idle server increases with increasing threshold values $Q, N \& M$. Figure 5 shows the impact of $\varphi$ on the expected queue length wherein for a fixed $\varphi$, the expected queue length decreases with increasing service rate $\mu$. 


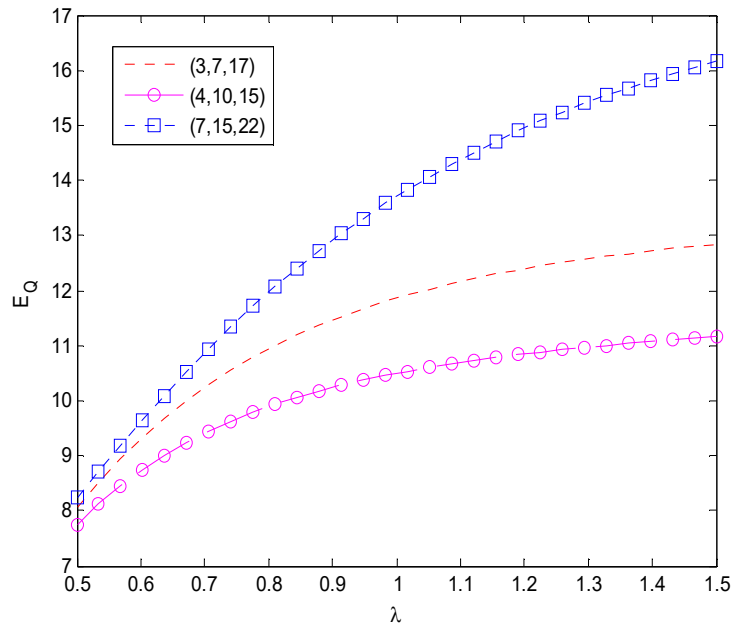

Figure 6. $\lambda$ versus $E_{Q}$

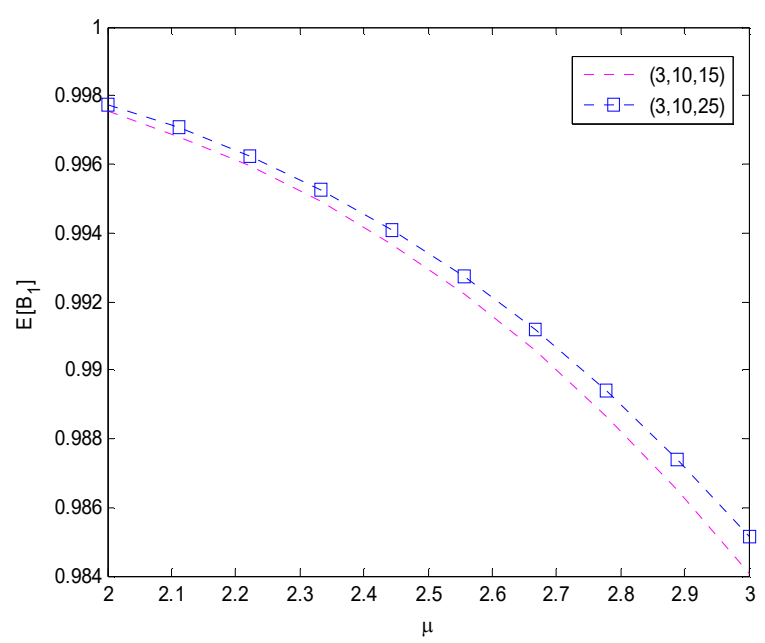

Figure 8. Effect of $\mu$ on $E\left[B_{1}\right]$

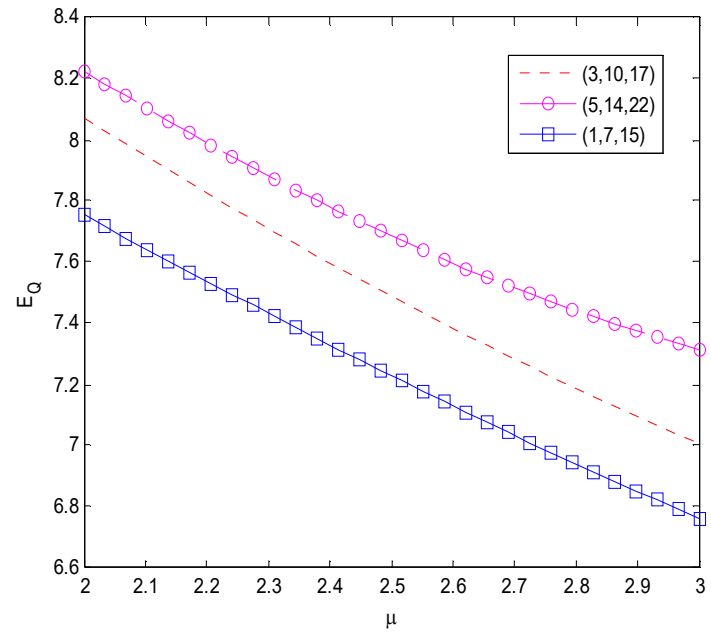

Figure 7. $\mu$ versus $E_{Q}$

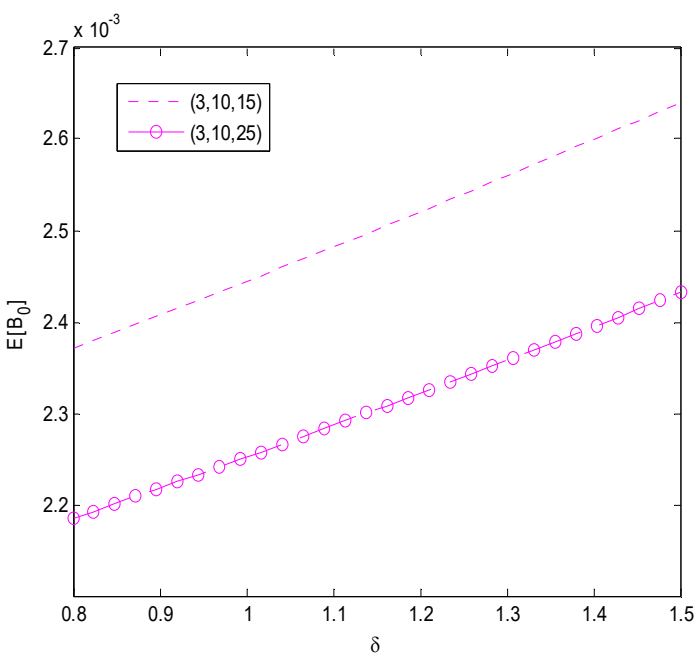

Figure 9. Effects of $\delta$ on $E\left[B_{0}\right]$

The impact of $\lambda$ on the expected queue length is illustrated in Figure 6 . As it expected in physical sense, the expected queue length increases with the increasing $\lambda$. Furthermore, for a fixed $\lambda$, the expected queue length increases with increasing threshold values $Q, N \& M$. Conversely, the expected queue length decreases with increasing $\mu$ in Figure 7 .

When a regular busy server provides a service with high service rate, failed machines will be repaired and depart the queue quickly. This eventually makes the queue empty and the expected number of busy server will decrease as server will quit to working vacations period. This fact is shown in Figure 8. The effect of $\delta$ on $E\left[B_{0}\right]$ with different threshold values $Q, N \& M$ is explained in Figure 9 .

\section{Conclusion}

In this paper, the performance analysis of $M / M / 2$ machine repair system with balking, reneging, multiple working vacations and triadic $(0, Q, N, M)$ policy has been carried out. A recursive method to find the steady state probabilities of the system has developed. It is observed that the recursive method is powerful and easy to implement. Various performance measures of the model such as expected queue length, expected number of busy servers, average balking rate, average reneging rate, etc., have been presented. Numerical illustrations to these performance measures have been discussed in figures form to authenticate the theoretical results. As a consequence, it is seen that the present model represents a machine repair system which is encountered in the physical world. The transient analysis of the present model is left for future research.

\section{Availability of data and materials}

Not applicable. 


\section{Competing interests}

The author would like to declare no conflict of interests.

\section{Funding}

Not applicable.

\section{Acknowledgements}

The author would like to express his sincere gratitude to the editors and reviewers of this manuscript.

\section{Reference}

Ahuja, A. and Jain, A. 2019. Joining strategies in multiple vacation machining system with heterogeneous servers. Compusoft, 8(7): 3269-3273.

Chen, W.L. and Wang, K.H. 2018. Reliability analysis of a retrial machine repair problem with warm standbys and a single server with N-policy. Reliability Engineering \& System Safety, 180: 476-486.

Chen, W.L., Wen, C.H. and Chen, Z.H. 2016. System reliability of a machine repair system with a multiplevacation and unreliable server. Journal of Testing and Evaluation, 44(4): 1745-1755.

He, G., Wu, W. and Zhang, Y. 2019. Performance analysis of machine repair system with single working vacation. Communications in Statistics-Theory and Methods, 48(22): 5602-5620.

Jain, M. and Meena, R.K. 2018. Vacation model for Markov machine repair problem with two heterogeneous unreliable servers and threshold recovery. Journal of Industrial Engineering International, 14(1): 143-152.

Jain, M., Shekhar, C. and Shukla, S. 2016. A time-shared machine repair problem with mixed spares under Npolicy. Journal of Industrial Engineering International, 12(2): 145-157.

Kumar, K., Jain, M. and Shekhar, C. 2018. Machine repair system with F-policy, two unreliable servers, and warm standbys. Journal of Testing and Evaluation, 47(1): 361-383.

Ma, M. and Zhao, B. 2016, May. Reliability Performance of Machine Repair Problem with Balking, Reneging, Warm spares and Synchronous Multiple Vacation. In 2016 International Conference on Applied Mathematics, Simulation and Modelling. Atlantis Press.

Meena, R.K., Jain, M., Sanga, S.S. and Assad, A. 2019. Fuzzy modeling and harmony search optimization for machining system with general repair, standby support and vacation. Applied Mathematics and Computation, 361: 858-873.

Ojobor, S.A. and Ogini, N.O. 2016. Machine Interference Problem with Reliable Server under Multiple Vacations Policy. Journal of Advances in Mathematics and Computer Science, 1-15.

Sharma, R. 2016, October. Threshold N-policy for machine interference problem with additional repairman and spares under bernoulli vacation schedule. In 2016 11th International Conference on Reliability, Maintainability and Safety (ICRMS) (pp. 1-5). IEEE.

Shekhar, C., Jain, M. and Raina, A.A. 2017. Transient analysis of machining system with spare provisioning and geometric reneging. International Journal of Mathematics in Operational Research, 11(1).

Shekhar, C., Jain, M., Raina, A. and Mishra, R. 2017. Sensitivity analysis of repairable redundant system with switching failure and geometric reneging. Decision Science Letters, 6(4): 337-350.

Wang, K.H., Chung, C.Y. and Yang, D.Y. 2015. Diffusion Approximation for G/G/R Machine Repair Problems with Balking and Reneging. Journal of Testing and Evaluation, 43(1): 179-190. 